\title{
Association between clinical risk factors and severity of dysphagia after extubation based on a videofluoroscopic swallowing study
}

\author{
Won-Jong Yang ${ }^{1,2}$, Eunhee Park ${ }^{1}$, Yu-Sun Min ${ }^{1,2}$, Jae-Won Huh ${ }^{3}$, Ae Ryoung Kim², , Hyun-Min Oh \\ Tae-Woo Nam ${ }^{1}$, and Tae-Du Jung,
}

\author{
${ }^{1}$ Department of Rehabilitation \\ Medicine, Kyungpook National \\ University Chilgok Hospital, Daegu; \\ ${ }^{2}$ Department of Rehabilitation \\ Medicine, School of Medicine, \\ Kyungpook National University, \\ Daegu; ${ }^{3}$ Department of \\ Rehabilitation Medicine, Kyungpook \\ National University Hospital, Daegu, \\ Korea
}

Received: February 8, 2018

Revised : July 18, 2018

Accepted: July 25, 2018

\section{Correspondence to}

Tae-Du Jung, M.D.

Department of Rehabilitation

Medicine, Kyungpook National

University Chilgok Hospital, 807

Hoguk-ro, Buk-gu, Daegu 41404,

Korea

Tel: +82-53-200-2167

Fax: $+82-53-200-2033$

E-mail: teedo522@knu.ac.kr
Background/Aims: This study aimed to evaluate the correlation between clinical risk factors of post-extubation dysphagia (PED) and the severity of impaired pharyngeal swallowing function assessed via videofluoroscopic swallowing studies (VFSSs).

Methods: This study was a retrospective review of medical records. Of 116 patients who were admitted to the intensive care unit and underwent VFSS, 32 who had non-neurologic disorders and experienced prolonged intubation (for more than 48 hours) were diagnosed with PED. The severity of PED was evaluated by using a functional dysphagia scale (FDS) and a penetration aspiration scale (PAS), on the basis of VFSS.

Results: The Simplified Acute Physiology Score 3 and total FDS score were positively correlated $(r=0.40, p=0.02)$. Intubation duration was positively correlated with total PAS and FDS scores $(r=0.62, p<0.001 ; r=0.65, p<0.001$, respectively). The amounts of residue in the valleculae (RV) and pyriform sinuses (RP) were associated with intubation duration $(r=0.58, p<0.001 ; r=0.57, p<0.001$, respectively). Multivariate regression analysis revealed that intubation duration was significantly associated with the total FDS score, RV and RP subscales of the FDS, and total PAS score.

Conclusions: The severity of impaired swallowing function, particularly the amount of residue in the pharyngeal recesses assessed via VFSS, was strongly associated with both severity of medical illness and intubation duration. Intubation duration could be a prognostic factor for assessing impaired swallowing function on the basis of VFSS.

Keywords: Post-extubation dysphagia; Videofluoroscopic swallowing study; Intubation, intratracheal; Deglutition disorders; Extubation

\section{INTRODUCTION}

Post-extubation dysphagia (PED) is defined as the inability or difficulty to safely and effectively swallow food and liquid after extubation of endotracheal tubes; it has been reported in $3 \%$ to $62 \%$ of individuals who underwent intubation $[1,2]$ and can lead to severe complications such as malnutrition, dehydration, aspiration, and pneumonia [3]. The maintenance of endotracheal tubes for mechanical ventilation could lead to focal mucosal 
ulcerations or the inflammation of pharyngeal or laryngeal structures [4]. In addition, prolonged oral endotracheal intubation, defined as intubation time longer than 48 hours $[2,5]$, could influence neuromuscular weakness of the oropharyngeal structures $[6,7]$.

Previous studies regarding PED examined patients who were admitted to the intensive care unit (ICU) and examined with a flexible laryngoscope [8-10]. This fiberoptic endoscopic evaluation could facilitate the visualization of pharyngeal, laryngeal, and vocal cord structures, as well as the detection of aspiration; however, it could not demonstrate critical events of pharyngeal swallowing, including contraction of the pharynx during aspiration. A commonly used assessment tool for dysphagia is the videofluoroscopic swallowing study (VFSS), which is highly sensitive for aspiration and facilitates the precise assessment of complex abnormalities throughout the swallowing process [11-13]. However, thus far, few studies have assessed the associations between clinical characteristics of PED and the severity of impaired swallowing function by using VFSS in patients with PED. Therefore, the aim of this study was to evaluate the associations between clinical risk factors for PED and the severity of impaired pharyngeal swallowing dysfunction, as determined by VFSS, in patients with PED.

\section{METHODS}

\section{Participants}

This study involved a retrospective review of the medical records of patients who were admitted to the ICU of Kyungpook National University Hospital and referred for VFSS from January 2013 to October 2017. By using bedside screening tests, a physician checked eligibility for inclusion on the basis of patient mental status by using the Glasgow Coma Scale (GCS), neurological examination, and assessment of cranial nerve function and symptoms of aspiration during swallowing. We included patients who experienced dysphagia after extubation, had a GCS score greater than 14, had a history of prolonged intubation (longer than 48 hours), and who were older than 18 years. The exclusion criteria were as follows: any preexisting underlying brain disease that may cause dysphagia, such as stroke, brain tumor, or Parkinson disease; preexisting cognition impairment; new-onset neurology symptoms, such as severe weakness or sensory changes; poor cooperation; tracheostomy state; or preexisting dysphagia.

This study was approved by the Institutional Review Board (IRB) of Kyungpook National University Hospital (IRB number 2018-01-031). Written informed consent by the patients was waived due to a retrospective nature of our study.

\section{Outcome measurements}

The primary endpoints of the study were the severity of dysphagia after prolonged intubation and the identification of clinical factors related to the severity of dysphagia. We reviewed the following data: diagnosis at the time of ICU admission, length of stay in the ICU, duration of intubation, size of the endotracheal tube, and timing of swallowing function assessment after extubation. In addition, the severity of clinical illness at the time of admission to the ICU was evaluated via the Simplified Acute Physiology Score 3 (SAPS 3) [14,15].

To analyze the clinical characteristics of patients with PED, on the basis of the medical condition for which endotracheal intubation was initiated, patients were categorized into three groups. Group 1 included patients who had infections or septic conditions; group 2 included patients who had a history of heart failure due to ischemic heart disease or valvular disease; group 3 included patients who were intubated after cardiothoracic surgery.

\section{Videofluoroscopic swallowing study}

A physician performed the videofluoroscopic swallowing examinations with an assistant, and the examination findings were reviewed by an expert in physical medicine and rehabilitation. We prepared diluted liquid barium according to the standard VFSS protocol guideline [16]. Thin liquid, thick liquid, soft food, and solid food were mixed with diluted barium. A syringe was used to feed thin and thick liquid. We selected porridge as the soft food and rice as the solid food; we used a spoon to feed soft and solid foods. To check for aspiration, a chest radiograph was acquired after the VFSS.

We evaluated the severity of dysphagia with the functional dysphagia scale (FDS) [16] and penetration aspiration scale (PAS) [17] on the basis of VFSS findings. The FDS, with a total score of 100 points, consists of 11 sub- 
scales and quantitatively evaluates swallowing function by using information from the VFSS (Supplementary Table 1). The PAS is an 8-point indicator for the assessment of airway invasion. The presence of airway penetration (entry of contrast material into the laryngeal vestibule) and aspiration (entry of contrast material below the level of the true vocal folds) were recorded for each swallow by using the PAS score (Supplementary Table 2).

\section{Statistical analyses}

Statistical analyses were performed using SPSS for Windows, version 13.0 (SPSS Inc., Chicago, IL, USA); $p$ values less than 0.05 were considered significant. Three groups, which were divided on the basis of diagnosis of critical illness, were compared by using the Kruskal-Wallis test. Multivariate regression was used to determine associations between individual clinical risk factors and severity of dysphagia. These variables were selected because their univariate analysis $p$ values were $<0.20$. To analyze the correlation of clinical risk factors with the severity of swallowing function impairment, a Pearson correlation analysis was performed.

\section{RESULTS}

Of 116 patients who were admitted to the ICU and referred for a VFSS, 32 had a non-neurologic critical illness for which they were intubated for a prolonged time (longer than 48 hours), and were subsequently diagnosed with PED; these patients were considered eligible for this study (Fig. 1). Table 1 shows the demographics and clinical features of the participants. The mean SAPS 3 score was 55.91; the mean duration of intubation was $189.82 \pm 109.82$ hours. All patients showed normal findings with regard to lip closure, bolus formation, residue in the oral cavity, oral transit time, triggering of pharyngeal swallow, nasal penetration, and pharyngeal transit time. However, residue in the valleculae (RV) was observed in all patients and residue in the pyriform sinus (RP) was observed in 31 patients (96.8\%). Coating of the pharyngeal wall after swallowing was observed in 26 patients (81.2\%). There were no statistically significant differences among the three groups. Fig. 2 shows the features of VFSS, on the basis of severity of PED.

Multivariate regression analysis revealed that the dura-

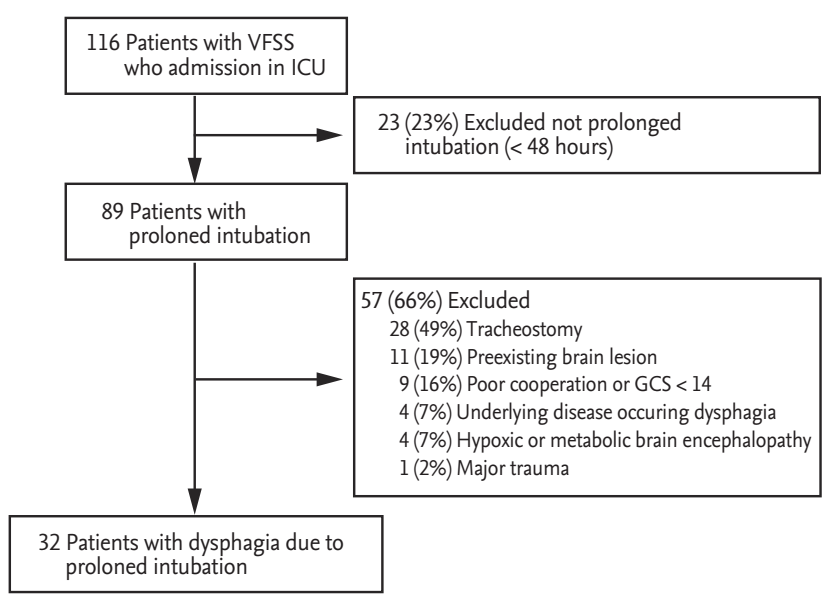

Figure 1. Flow diagram showing the subject selection process with the applied inclusion and exclusion criteria. VFSS, videofluoroscopic swallowing study; ICU, intensive care unit; GCS, Glasgow Coma Scale.

tion of intubation was significantly associated with PAS and FDS; moreover, the SAPS 3 score was significantly associated with the RV subset of FDS (Tables 2 and 3). The Pearson correlation test was performed to identify correlations among the indicators used in the present study (Table 4). There was a strong correlation between intubation duration and PAS score $(r=0.62, p<0.001)$. In addition, there was a strong correlation between intubation duration and FDS score $(r=0.65, p \leq 0.001)$; the RV and RP subscales of the FDS were correlated with intubation duration $(r=0.58, p<0.001 ; r=0.57, p<0.001$, respectively). Among the ICU-related clinical factors, the $\mathrm{SAPS}_{3}$ and FDS scores were correlated $(r=0.40, p=0.02)$. The SAPS 3 score was strongly correlated with the RV subset of the FDS $(r=0.50, p \leq 0.001)$. There were no significant correlations among the following factors: age, ICU admission duration, endotracheal tube size, timing of swallowing function assessment after extubation, and severity of swallowing function impairment.

\section{DISCUSSION}

This study aimed to evaluate the correlation between clinical risk factors of PED and the severity of impaired pharyngeal swallowing function through assessment by VFSS. The results of this study suggested that intubation duration was the most important clinical factor 
Table 1. Demographics and presenting clinical features of the participants

\begin{tabular}{|c|c|c|c|c|c|}
\hline \multirow{2}{*}{ Variable } & \multirow{2}{*}{ Total $(n=32)$} & \multicolumn{3}{|c|}{ Categorized groups } & \multirow{2}{*}{$p$ value } \\
\hline & & Group $1(n=9)$ & Group $2(n=12)$ & Group $3(n=11)$ & \\
\hline Age & $71.41 \pm 8.34$ & $69.01 \pm 10.00$ & $72.5 \pm 8.94$ & $77.0 \pm 8.34$ & 0.117 \\
\hline Sex & & & & & 0.362 \\
\hline Female & $15(46.9)$ & $3(33 \cdot 3)$ & $5(41.7)$ & $7(63.6)$ & \\
\hline Male & $17(53.1)$ & $6(66.7)$ & $7(58.3)$ & $4(36.4)$ & \\
\hline Length of stay in ICU, hr & $457.03 \pm 362.19$ & $364.6 \pm 314.5$ & $462.2 \pm 381.9$ & $527.1 \pm 392.4$ & 0.329 \\
\hline $\mathrm{SAPS}_{3}$ & $55.91 \pm 12.28$ & $58.1 \pm 10.8$ & $55 \cdot 7 \pm 16.1$ & $54.4 \pm 9.1$ & 0.510 \\
\hline Duration of intubation, hr & $189.82 \pm 109.82$ & $199 \cdot 3 \pm 108.7$ & $178.5 \pm 112.2$ & $194.4 \pm 117.6$ & 0.792 \\
\hline Size of endotracheal tube, Fr & $7.47 \pm 0.25$ & $7 \cdot 5 \pm 0$ & $7 \cdot 5 \pm 0.3$ & $7.41 \pm 0.3$ & 0.293 \\
\hline TAE, day & $13.19 \pm 9.59$ & $10.0 \pm 6.1$ & $12.7 \pm 11.7$ & $16.4 \pm 9.2$ & 0.139 \\
\hline PAS & $5.06 \pm 2.41$ & $5.56 \pm 2.19$ & $5.33 \pm 2.64$ & $4.36 \pm 2.38$ & 0.426 \\
\hline FDS & $38.25 \pm 8.93$ & $42.67 \pm 4.24$ & $38.00 \pm 9.46$ & $34.91 \pm 10.21$ & 0.425 \\
\hline RV & $9.88 \pm 3.21$ & $11.56 \pm 1.33$ & $9.33 \pm 3.55$ & $9.09 \pm 3.62$ & 0.466 \\
\hline $\mathrm{RP}$ & $8.62 \pm 3.23$ & $8.89 \pm 3.33$ & $8.33 \pm 3.89$ & $4.67 \pm 4.67$ & 0.256 \\
\hline $\mathrm{CP}$ & $8.12 \pm 3.97$ & $10.67 \pm 2.00$ & $8.33 \pm 3.17$ & $7.27 \pm 3.50$ & 0.637 \\
\hline
\end{tabular}

Values are presented as mean \pm SD or number $(\%)$.

ICU, intensive care unit; SAPS 3, Simplified Acute Physiology Score 3; TAE, timing of swallowing function assessment after extubation; PAS, penetration aspiration scale; FDS, functional dysphagia scale; RV, residue in the valleculae; RP, residue in the pyriform sinus; CP, coating of the pharyngeal wall.
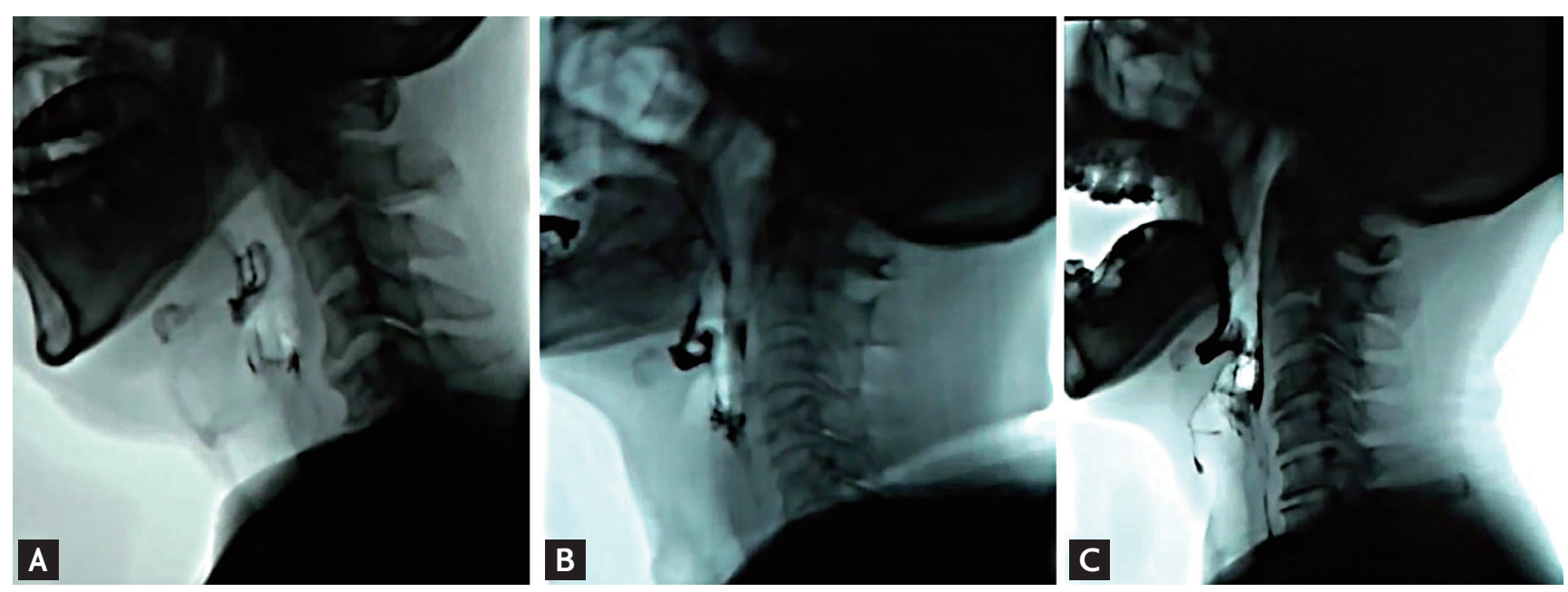

Figure 2. Features of videofluoroscopic swallowing studies according to the severity of post-extubation dysphagia (PED). (A) In a PED patient with functional dysphagia scale (FDS) 20 and penetration aspiration scale (PAS) 2, there is less than $10 \%$ residue in valleculae and pyriform sinus without coating of the pharyngeal wall. (B) In a PED patient with FDS 38 and PAS 4 , there is coating of the pharyngeal wall, with $50 \%$ residue in valleculae and pyriform sinus. (C) In a PED patient with FDS 46 and PAS 7 , there is aspiration and coating of the pharyngeal wall and valleculae and pyriform sinus have more than $50 \%$ residue.

associated with the severity of PED, followed by the SAPS 3 score. Our study demonstrated the clinical characteristics of PED on the basis of the medical condition for which endotracheal intubation was initiated, and showed quantification of dysfunction in the oral and laryngeal swallowing phases by using VFSS. 
Table 2. Results of univariate regression analysis of PAS, FDS, and the subgroups of FDS

\begin{tabular}{|c|c|c|c|c|c|c|c|c|}
\hline \multirow{3}{*}{ Variable } & \multirow{2}{*}{\multicolumn{2}{|c|}{ PAS }} & \multicolumn{6}{|c|}{ FDS } \\
\hline & & & \multicolumn{2}{|c|}{ Total } & \multicolumn{2}{|c|}{$\mathrm{RV}$} & \multicolumn{2}{|c|}{$\mathrm{RP}$} \\
\hline & $\beta$ & $p$ value & $\beta$ & $p$ value & $\beta$ & $p$ value & $\beta$ & $p$ value \\
\hline Age & 0.03 & 0.574 & -0.05 & 0.79 & 0.01 & 0.898 & -0.07 & 0.305 \\
\hline Length of stay in ICU, hr & 0.00 & 0.08 & 0.01 & 0.14 & 0.00 & 0.313 & 0.00 & 0.329 \\
\hline $\mathrm{SAPS}_{3}$ & 0.02 & 0.528 & 0.29 & 0.023 & 0.13 & 0.003 & 0.04 & 0.43 \\
\hline Duration of intubation, hr & 0.01 & $<0.001$ & 0.05 & $<0.001$ & 0.02 & $<0.001$ & 0.02 & 0.001 \\
\hline Size of endotracheal tube, Fr & 1.05 & 0.55 & 7.24 & 0.262 & 1.97 & 0.398 & 0.32 & 0.893 \\
\hline TAE, day & -0.04 & 0.395 & -0.11 & 0.512 & -0.06 & 0.314 & -0.04 & 0.482 \\
\hline
\end{tabular}

PAS, penetration aspiration scale; FDS, functional dysphagia scale; RV, residue in the valleculae; RP, residue in the pyriform sinus; ICU, intensive care unit; SAPS 3 , Simplified Acute Physiology Score 3; TAE, timing of swallowing function assessment after extubation.

Table 3. Results of multivariate regression analysis of PAS, FDS, and the subgroups of FDS

\begin{tabular}{|c|c|c|c|c|c|}
\hline Variable & Estimate & Standardized & $\mathrm{SE}$ & $t$ value & $\operatorname{Pr}(>|t|)$ \\
\hline \multicolumn{6}{|l|}{ PAS } \\
\hline Intercept & 2.497 & 0.000 & 0.679 & 3.679 & 0.001 \\
\hline Length of stay in ICU, hr & -0.002 & -0.254 & 0.001 & -1.266 & 0.216 \\
\hline Duration of intubation, hr & 0.018 & 0.802 & 0.004 & 3.996 & $<0.001$ \\
\hline \multicolumn{6}{|l|}{ Total FDS } \\
\hline Intercept & 17.893 & 0.000 & $5 \cdot 588$ & 3.202 & 0.003 \\
\hline Length of stay in ICU, hr & -0.007 & -0.277 & 0.005 & -1.492 & 0.147 \\
\hline SAPS $_{3}$ & 0.195 & 0.268 & 0.096 & 2.031 & 0.052 \\
\hline Duration of intubation, hr & 0.066 & 0.816 & 0.015 & $4 \cdot 411$ & $<0.001$ \\
\hline \multicolumn{6}{|l|}{ RV of FDS } \\
\hline Intercept & 0.509 & 0.000 & 1.962 & 0.259 & 0.797 \\
\hline $\mathrm{SAPS}_{3}$ & 0.115 & 0.439 & 0.033 & $3 \cdot 427$ & 0.002 \\
\hline Duration of intubation, hr & 0.016 & 0.532 & 0.004 & 4.157 & $<0.001$ \\
\hline \multicolumn{6}{|l|}{ RP of FDS } \\
\hline Intercept & $5 \cdot 426$ & 0.000 & 0.962 & 5.643 & $<0.001$ \\
\hline Duration of intubation, hr & 0.017 & 0.573 & 0.004 & 3.828 & 0.001 \\
\hline
\end{tabular}

PAS, penetration aspiration scale; FDS, functional dysphagia scale; SE, standard error; ICU, intensive care unit; SAPS 3 , Simplified Acute Physiology Score 3; RV, residue in the valleculae; RP, residue in the pyriform sinus.

Previous studies have identified many clinical factors associated with PED, although the results of those studies were controversial [2]; the use of fiberoptic endoscopic evaluations in patients with PED revealed that intubation duration was related to the incidence and severity of PED $[1,2,18,19]$. In our study, we noted a correlation between the severity of PED, especially the amount of RV, and the severity of clinical illness, similar to the findings of another study [20]. Post-swallowing vallecular residue is significantly associated with penetration and aspiration in dysphagia [21]. These results supported those of previous studies, which concluded that scoring systems of critically ill states, such as the Acute Physiology and Chronic Health Evaluation and the Sequential Organ Failure Assessment, can serve as predictors of ventilator-associated pneumonia [22,23]. 
Table 4. Results of the Pearson correlation test: correlations among age, ICU-related parameters, and measurement of dysphagia

\begin{tabular}{|c|c|c|c|c|c|c|c|c|}
\hline \multirow{3}{*}{ Variable } & \multirow{2}{*}{\multicolumn{2}{|c|}{ PAS }} & \multicolumn{6}{|c|}{ FDS } \\
\hline & & & \multicolumn{2}{|c|}{ Total } & \multicolumn{2}{|c|}{ RV } & \multicolumn{2}{|c|}{$\mathrm{RP}$} \\
\hline & $r$ & $p$ value & $r$ & $p$ value & $r$ & $p$ value & $r$ & $p$ value \\
\hline Age & 0.10 & 0.57 & -0.05 & 0.79 & 0.02 & 0.90 & -0.19 & 0.31 \\
\hline Length of stay in ICU, hr & 0.31 & 0.08 & 0.27 & 0.14 & 0.18 & 0.31 & 0.18 & 0.33 \\
\hline $\mathrm{SAPS}_{3}$ & 0.12 & 0.53 & 0.40 & 0.02 & 0.50 & $<0.001$ & 0.14 & 0.43 \\
\hline Duration of intubation, hr & 0.62 & $<0.001$ & 0.65 & $<0.001$ & 0.58 & $<0.001$ & 0.57 & $<0.001$ \\
\hline Size of endotracheal tube, Fr & 0.11 & 0.55 & 0.20 & 0.26 & 0.15 & 0.40 & 0.02 & 0.89 \\
\hline TAE, day & -0.16 & 0.40 & -0.12 & 0.51 & -0.18 & 0.31 & -0.13 & 0.48 \\
\hline
\end{tabular}

ICU, intensive care unit; PAS, penetration aspiration scale; FDS, functional dysphagia scale; RV, residue in the valleculae; RP, residue in the pyriform sinus; SAPS 3 , Simplified Acute Physiology Score 3; TAE, timing of swallowing function assessment after extubation.

Our study and other previous studies have shown that endotracheal tube size is not associated with PED $[1,5]$. These results are not consistent with the hypothesis of another study, which stated that endotracheal tube size could affect the severity of PED [24]. However, in our study, the results of endotracheal tube size could not be generalized because the endotracheal tubes used in most patients were similar in size.

Previous studies have suggested that age may significantly affect PED [9,25]. Barquist et al. [26] reported that PED was more severe in patients older than 55 years. In our study, patient age did not correlate with the severity of PED. However, this observation may be because of the inclusion of elderly patients. Prolonged intubation has been shown to reduce tongue strength and endurance [27]. However, in the present study, the patients demonstrated normal findings with respect to bolus formation, presence of residue in the oral cavity, and oral transit time. Most patients with PED exhibited residue in the pharyngeal recesses, such as the valleculae and pyriform sinus, which indicated that prolonged intubation could cause weakness of the laryngeal muscles.

The findings of our study suggest that compared with dysphagia caused by other diseases, PED involves different features and mechanisms. Dysfunction of lip closure and bolus formation, residue formation in the oral cavity, prolonged oral transit time and pharyngeal transit time, and triggering of pharyngeal swallow were not observed in PED cases. Clinical findings of PED differed from those of dysphagia due to neurological causes, such as stroke, or anatomical causes, such as laryngeal cancer $[28,29]$. Our study also showed no correlation between the duration of endotracheal intubation and coating of the pharyngeal wall. This result is similar to the finding of a study by Stauffer et al. [30], which showed no correlation between the duration of endotracheal intubation in intubated patients and the severity of laryngeal lesions. These results support a mechanism in which laryngeal lesions are caused by direct mucosal injury and inflammation during intubation [7].

The presence of moderate or severe dysphagia was significantly associated with the composite outcome of pneumonia, reintubation, and death [1]. Further, patients with PED also exhibit a high prevalence of pneumonia, high mortality, and poor outcomes $[1,31]$. Thus, a rehabilitation team that administers special interventions to patients with PED is necessary. However, little is known regarding specific interventions for PED. According to a randomized control study, preemptive swallowing electrical stimulation during intubation can lead to recovery of swallowing function after prolonged intubation [32]. Because RV and RP both influence aspiration in patients with $\mathrm{PED}$, pharyngeal weakness should be focused more specifically on PED rehabilitation. Several studies have shown that Shaker exercise, effortful swallowing, and tongue-to-palate resistance training can reduce the amount of residue in the pharynx and improve pharyngeal muscle contraction in patients with dysphagia [33-36]. These exercises for dysphagia may reduce the occurrence of aspiration by reducing post-swallowing residue in the pharyngeal recesses of patients with PED. However, further research is warranted to identify spe- 
cific rehabilitative interventions for PED.

Our study has a few limitations. First, this study included a heterogeneous patient population with various underlying diseases, because we included patients admitted to all ICUs in the hospital. To compare the characteristics of PED due to various underlying diseases, the patients were divided into three groups on the basis of underlying disease; however, there were no significant differences among the three groups. Second, the small sample size of our study limits the overall reliability of our analysis and may reduce the clinical impact of the results. Third, because of the cross-sectional design of this study, the prognosis and mortality of the participants could not be confirmed. Thus, an additional longitudinal study is warranted. Finally, we could not exclude patients with critical illness polyneuropathy and myopathy, which might influence swallowing function because the patients enrolled in this study were not evaluated for critical illness polyneuropathy and myopathy. However, there have been no studies of the prevalence of dysphagia in patients with critical illness polyneuropathy [37], and it is difficult to distinguish the cause of dysphagia in these patients because most critical illness polyneuropathy patients have a history of intubation. Critical illness polyneuropathy is suspected to occur when mechanical ventilation has been performed for more than 7 days and is diagnosed through electromyography (EMG) and physical examination [38]. In a prospective cohort study about EMG assessment of ICU patients without neuromuscular disease, the incidence of critical illness polyneuropathy and myopathy was $25.3 \%$ in patients who underwent mechanical ventilation for more than 7 days [39]. Future prospective studies on patients with PED should exclude the possibility of critical illness polyneuropathy and myopathy using EMG.

In conclusion, this study shows that the severity of dysphagia is most strongly correlated with the duration of oral endotracheal intubation and that the SAPS 3 score is positively correlated with dysphagia severity. Patients who have undergone longer intubation duration or have higher SAPS 3 scores should be evaluated for dysphagia after extubation to ensure more appropriate interventions for dysphagia.

\section{KEY MESSAGE}

1. Intubation duration was the most important factor associated with the severity of post-extubation dysphagia.

2. The severity of post-extubation dysphagia was also associated with the Simplified Acute Physiology Score 3 (SAPS 3) score.

3. Patients who have been intubated for a long duration and patients with high SAPS 3 scores should be thoroughly examined for dysphagia after extubation, to ensure that timely, appropriate interventions can be administered.

\section{Conflict of interest}

No potential conflict of interest relevant to this article was reported.

\section{REFERENCES}

1. Macht M, Wimbish T, Clark BJ, et al. Postextubation dysphagia is persistent and associated with poor outcomes in survivors of critical illness. Crit Care 2011;15:R231.

2. Skoretz SA, Flowers HL, Martino R. The incidence of dysphagia following endotracheal intubation: a systematic review. Chest 2010;137:665-673.

3. Rofes L, Arreola V, Almirall J, et al. Diagnosis and management of oropharyngeal dysphagia and its nutritional and respiratory complications in the elderly. Gastroenterol Res Pract 2011;2011:818979.

4. Benjamin B, Holinger LD. Laryngeal complications of endotracheal intubation. Ann Otol Rhinol Laryngol 2008;117(9 Suppl):2-20.

5. Kwok AM, Davis JW, Cagle KM, Sue LP, Kaups KL. Post-extubation dysphagia in trauma patients: it's hard to swallow. Am J Surg 2013;206:924-927.

6. Macht M, Wimbish T, Bodine C, Moss M. ICU-acquired swallowing disorders. Crit Care Med 2013;41:2396-2405.

7. Tolep K, Getch CL, Criner GJ. Swallowing dysfunction in patients receiving prolonged mechanical ventilation. Chest 1996;109:167-172.

8. Ajemian MS, Nirmul GB, Anderson MT, Zirlen DM, Kwasnik EM. Routine fiberoptic endoscopic evaluation of swallowing following prolonged intubation: implications 
for management. Arch Surg 2001;136:434-437.

9. El Solh A, Okada M, Bhat A, Pietrantoni C. Swallowing disorders post orotracheal intubation in the elderly. Intensive Care Med 2003;29:1451-1455.

10. Leder SB, Cohn SM, Moller BA. Fiberoptic endoscopic documentation of the high incidence of aspiration following extubation in critically ill trauma patients. Dysphagia 1998;13:208-212.

11. Eisenhuber E, Schima W, Schober E, et al. Videofluoroscopic assessment of patients with dysphagia: pharyngeal retention is a predictive factor for aspiration. AJR Am J Roentgenol 2002;178:393-398.

12. Bours GJ, Speyer R, Lemmens J, Limburg M, de Wit R. Bedside screening tests vs. videofluoroscopy or fibreoptic endoscopic evaluation of swallowing to detect dysphagia in patients with neurological disorders: systematic review. J Adv Nurs 2009;65:477-493.

13. Kim J, Oh BM, Kim JY, Lee GJ, Lee SA, Han T'R. Validation of the videofluoroscopic dysphagia scale in various etiologies. Dysphagia 2014;29:438-443.

14. Metnitz PG, Moreno RP, Almeida E, et al. SAPS 3: from evaluation of the patient to evaluation of the intensive care unit. Part 1: objectives, methods and cohort description. Intensive Care Med 2005;31:1336-1344.

15. Moreno RP, Metnitz PG, Almeida E, et al. SAPS 3: from evaluation of the patient to evaluation of the intensive care unit. Part 2: development of a prognostic model for hospital mortality at ICU admission. Intensive Care Med 2005;31:1345-1355.

16. Han TR, Paik NJ, Park JW. Quantifying swallowing function after stroke: a functional dysphagia scale based on videofluoroscopic studies. Arch Phys Med Rehabil 2001;82:677-682.

17. Rosenbek JC, Robbins JA, Roecker EB, Coyle JL, Wood JL. A penetration-aspiration scale. Dysphagia 1996;11:93-98.

18. Barker J, Martino R, Reichardt B, Hickey EJ, Ralph-Edwards A. Incidence and impact of dysphagia in patients receiving prolonged endotracheal intubation after cardiac surgery. Can J Surg 2009;52:119-124.

19. Oliveira ACM, Friche AAL, Salomao MS, Bougo GC, Vicente LCC. Predictive factors for oropharyngeal dysphagia after prolonged orotracheal intubation. Braz J Otorhinolaryngol 2018;84:722-728.

20. Kim MJ, Park YH, Park YS, Song YH. Associations between prolonged intubation and developing post-extubation dysphagia and aspiration pneumonia in non-neuro- logic critically ill patients. Ann Rehabil Med 2015;39:763771.

21. Molfenter SM, Steele CM. The relationship between residue and aspiration on the subsequent swallow: an application of the normalized residue ratio scale. Dysphagia 2013;28:494-500.

22. Froon AH, Bonten MJ, Gaillard CA, et al. Prediction of clinical severity and outcome of ventilator-associated pneumonia. Comparison of simplified acute physiology score with systemic inflammatory mediators. Am J Respir Crit Care Med 1998;158:1026-1031.

23. Gursel G, Demirtas S. Value of APACHE II, SOFA and CPIS scores in predicting prognosis in patients with ventilator-associated pneumonia. Respiration 2006;73:503508.

24. Rassameehiran S, Klomjit S, Mankongpaisarnrung C, Rakvit A. Postextubation dysphagia. Proc (Bayl Univ Med Cent) 2015;28:18-20.

25. Hogue CW Jr, Lappas GD, Creswell LL, et al. Swallowing dysfunction after cardiac operations. Associated adverse outcomes and risk factors including intraoperative transesophageal echocardiography. J Thorac Cardiovasc Surg 1995;110:517-522.

26. Barquist E, Brown M, Cohn S, Lundy D, Jackowski J. Postextubation fiberoptic endoscopic evaluation of swallowing after prolonged endotracheal intubation: a randomized, prospective trial. Crit Care Med 2001;29:17101713.

27. Park HS, Koo JH, Song SH. Association of post-extubation dysphagia with tongue weakness and somatosensory disturbance in non-neurologic critically ill patients. Ann Rehabil Med 2017;41:961-968.

28. Martino R, Foley N, Bhogal S, Diamant N, Speechley M, Teasell R. Dysphagia after stroke: incidence, diagnosis, and pulmonary complications. Stroke 2005;36:2756-2763.

29. Nguyen NP, Frank C, Moltz CC, et al. Impact of dysphagia on quality of life after treatment of head-and-neck cancer. Int J Radiat Oncol Biol Phys 2005;61:772-778.

30. Stauffer JL, Olson DE, Petty TL. Complications and consequences of endotracheal intubation and tracheotomy. A prospective study of 150 critically ill adult patients. Am J Med 1981;70:65-76.

31. Schefold JC, Berger D, Zurcher P, et al. Dysphagia in mechanically ventilated ICU patients (DYnAMICS): a prospective observational trial. Crit Care Med 2017;45:20612069. 
32. Hwang $\mathrm{CH}$, Choi $\mathrm{KH}$, Ko YS, Leem CM. Pre-emptive swallowing stimulation in long-term intubated patients. Clin Rehabil 2007;21:41-46.

33. Shaker R, Easterling C, Kern M, et al. Rehabilitation of swallowing by exercise in tube-fed patients with pharyngeal dysphagia secondary to abnormal UES opening. Gastroenterology 2002;122:1314-1321.

34. Logemann JA, Rademaker A, Pauloski BR, et al. A randomized study comparing the Shaker exercise with traditional therapy: a preliminary study. Dysphagia 2009;24:403-411.

35. Jang HJ, Leigh JH, Seo HG, Han TR, Oh BM. Effortful swallow enhances vertical hyolaryngeal movement and prolongs duration after maximal excursion. J Oral Rehabil 2015;42:765-773.
36. Kim HD, Choi JB, Yoo SJ, Chang MY, Lee SW, Park JS. Tongue-to-palate resistance training improves tongue strength and oropharyngeal swallowing function in subacute stroke survivors with dysphagia. J Oral Rehabil 2017;44:59-64.

37. Ponfick M, Linden R, Nowak DA. Dysphagia: a common, transient symptom in critical illness polyneuropathy: a fiberoptic endoscopic evaluation of swallowing study. Crit Care Med 2015;43:365-372.

38. Latronico N, Bolton CF. Critical illness polyneuropathy and myopathy: a major cause of muscle weakness and paralysis. Lancet Neurol 2011;10:931-941.

39. De Jonghe B, Sharshar T, Lefaucheur JP, et al. Paresis acquired in the intensive care unit: a prospective multicenter study. JAMA 2002;288:2859-2867. 
KJIM'

The Korean Journal of Internal Medicine Vol. 35, No. 1, January 2020

Supplementary Table 1. Functional dysphagia scale using videofluoroscopic swallowing study in stroke patients

\begin{tabular}{|c|c|c|}
\hline Factor & Coded value & Score \\
\hline \multirow[t]{3}{*}{ Lip closure } & Intact & o \\
\hline & Inadequate & 5 \\
\hline & None & 10 \\
\hline \multirow[t]{3}{*}{ Bolus formation } & Intact & 0 \\
\hline & Inadequate & 3 \\
\hline & None & 6 \\
\hline \multirow[t]{4}{*}{ Residue in oral cavity } & None & 0 \\
\hline & $\leq 10 \%$ & 2 \\
\hline & $10 \%-50 \%$ & 4 \\
\hline & $\geq 50 \%$ & 6 \\
\hline \multirow[t]{2}{*}{ Oral transit time } & $\leq 1.5 \mathrm{sec}$ & O \\
\hline & $\geq 1.5 \mathrm{sec}$ & 6 \\
\hline \multirow[t]{2}{*}{ Triggering of pharyngeal swallow } & Normal & O \\
\hline & Delayed & 10 \\
\hline \multirow[t]{2}{*}{ Larynegal elevation and epiglottic closure } & Normal & 0 \\
\hline & Reduced & 12 \\
\hline \multirow[t]{4}{*}{ Nasal penetration } & None & o \\
\hline & $\leq 10 \%$ & 4 \\
\hline & $10 \%-50 \%$ & 8 \\
\hline & $\geq 50 \%$ & 12 \\
\hline \multirow[t]{4}{*}{ Residue in valleculae } & None & o \\
\hline & $\leq 10 \%$ & 4 \\
\hline & $10 \%-50 \%$ & 8 \\
\hline & $\geq 50 \%$ & 12 \\
\hline \multirow[t]{4}{*}{ Residue in pyriform sinuses } & None & o \\
\hline & $\leq 10 \%$ & 4 \\
\hline & $10 \%-50 \%$ & 8 \\
\hline & $\geq 50 \%$ & 12 \\
\hline \multirow[t]{2}{*}{ Coating of pharyngeal wall after swallow } & No & o \\
\hline & Yes & 10 \\
\hline \multirow[t]{2}{*}{ Pharyngeal transit time } & $\leq 1.0 \mathrm{sec}$ & 0 \\
\hline & $\geq 1.0 \mathrm{sec}$ & 4 \\
\hline Total & & 100 \\
\hline
\end{tabular}


Yang WJ, et al. Correlation of clinical factors and PED

Supplementary Table 2. Penetration aspiration scale

\begin{tabular}{lcl}
\hline Category & Score & \multicolumn{1}{c}{ Descriptions } \\
\hline No penetration or aspiration & 1 & Contrast does not enter the airway \\
Penetration & 2 & Contrast enters the airway, remains above vocal folds; no residue \\
& 3 & Contrast remains above vocal folds; visible residue remains \\
& 4 & Contrast contacts vocal folds; no residue \\
& 5 & Contrast contacts vocal folds; visible residue remains \\
& 6 & Contrast passes glottis; no subglottic residue visible \\
Aspiration & 7 & Contrast passes glottis; visible subglottic residue despite patient's response \\
& 8 & Contrast passes glottis; visible subglottic residue ; absent patient response \\
\hline
\end{tabular}

